ONLINE MUTATION REPORT

\title{
Characterisation of a novel TSC2 missense mutation in the GAP related domain associated with minimal clinical manifestations of tuberous sclerosis
}

\author{
K Mayer, M Goedbloed, K van Ziil, M Nellist, H-D Rott
}

J Med Genet 2004;41:e64 (http://www.jmedgenet.com/cgi/content/full/41/5/e64). doi: 10.1136/jmg.2003.010835

$\mathrm{T}$ uberous sclerosis (TSC) is an autosomal dominant disorder caused by mutations in either the TSCl or TSC2 gene. Both genes are tumour suppressor genes and encode the proteins hamartin and tuberin, respectively. Recent work has established that tuberin and hamartin interact to form a complex, and that this tuberin-hamartin complex antagonises signal transduction by preventing the activation of p70 S6 kinase, either directly or through mTOR..$^{1-3}$ The inhibition of p70 S6 kinase activity results in the dephosphorylation of ribosomal protein S6 and consequently leads to the down-regulation of cell growth. ${ }^{45}$

The clinical manifestations of TSC vary considerably between and within families. The phenotypic spectrum ranges from minor, asymptomatic skin affections, such as hypomelanotic macules, to drug resistant epilepsy, mental retardation, and increased morbidity due to cardiac tumours, cerebral astrocytomas, renal insufficiency, and lung affections. ${ }^{6}$ Although some studies suggest that TSCl mutations are more often associated with less severe disease, considerable overlap exists in the phenotypic spectrum associated with TSC1 and TSC2 mutations. ${ }^{7-9}$

Mutations that lead to truncation of tuberin or hamartin are clearly inactivating. However, nucleotide changes that result in amino acid substitutions may be either pathogenic or, alternatively, polymorphisms that do not disrupt tuberin or hamartin function and do not cause TSC. Investigations into the effect of amino acid substitutions on tuberin can help identify important functional domains and residues, and indicate whether putative mutations are likely to be pathogenic, either by disrupting the tuberin-hamartin complex, ${ }^{10}{ }^{11}$ or by preventing the inhibition of S6 phosphorylation. ${ }^{12}$

We report on a family with mild signs of TSC, segregating a novel missense mutation in exon 36 of the TSC2 gene that affects tuberin function. Although the entire GAP related domain of tuberin seems to be a target for missense mutations, ${ }^{13}$ only few changes have been reported so far in TSC2 exon 36, and none of these has so far been characterised for effect on tuberin function in relation to the clinical phenotype.

In this family, where the mild phenotype made a clinical diagnosis difficult, the clear effects on tuberin function of the amino acid substitution demonstrate how functional assays can help confirm the diagnosis of TSC, particularly if clinical and genetic analyses are not unequivocal.

\section{METHODS}

Mutation analysis and genotyping

Genomic DNA was extracted from peripheral lymphocytes by standard methods. All 21 coding exons of the TSC1 gene and all 41 coding exons of the TSC2 gene, including flanking intronic sequences, were amplified by PCR. Both strands were sequenced using a direct dideoxy method (ABI Prism

\section{Key points}

- Tuberous sclerosis (TSC) is an autosomal dominant disorder with a broad phenotypic spectrum. The clinical manifestations of the disease can vary considerably, both between and within families.

- Here we describe a family segregating a novel missense mutation, 4684G $>A$ (G1556S), in exon 36 of the TSC2 gene. Only the index patient fulfilled the present diagnostic criteria for TSC. However, clinical signs associated with TSC but insufficient for a definitive diagnosis were identified in additional family members carrying the same substitution.

- The mutation leads to a change of a highly conserved glycine residue in the GAP related domain of tuberin. To obtain additional evidence that the TSC2 4684G $>$ A mutation was the pathogenic mutation in this family, the effect of the G1556S substitution on tuberin function was investigated.

- Compared to wild type tuberin, the G1556S variant was hypophosphorylated, showed a reduced ability to form a complex with hamartin, and was unable to inhibit the phosphorylation of ribosomal protein S6, supporting the conclusion that the TSC2 4684G $>$ A substitution was the pathogenic mutation in this family. The implications of these results for the diagnosis of TSC patients are discussed.

Big Dye Terminator ${ }^{\mathrm{TM}}$, PE Applied Biosystems, Foster City, CA, USA) and analysed on an ABI3100 sequencer. Confirmation of sequence variants as well as haplotyping of unrelated controls was performed by RFLP analysis and Pyrosequencing $^{\mathrm{TM}}$ (www.pyrosequencing.com).

Linkage analysis was performed using the microsatelite markers D9S2126, D9S1830, D9S1199, and D9S1198 for TSC1, and KG8, 16AC2.5, and SM7 for TSC2.

\section{Functional analysis}

The full-length TSC1 and TSC2 expression constructs have been described previously. ${ }^{14}$ The TSC2 G1556S variant expression constructs were obtained by site directed mutagenesis of the original wild type TSC2 expression constructs using the Stratagene QuickChange kit (La Jolla, CA, USA). An expression construct encoding an activated $\mathrm{PKB}$ isoform was purchased from Upstate Biotechnology (www.upstate. edu/biochem). All the constructs used were sequenced completely. The tuberin specific and hamartin specific

Abbreviation: TSC, tuberous sclerosis complex 
antibodies used in this study have been described previously..$^{14}$ Phosphospecific antibodies against S6 (S235/236) and the PKB target sequence $((\mathrm{R} / \mathrm{K}) \mathrm{X}(\mathrm{R} / \mathrm{K}) \mathrm{XX}(\mathrm{T}-\mathrm{P} / \mathrm{S}-\mathrm{P}))$ were purchased from Cell Signaling Technology (www.cellsignal. com); antibodies against the polyhistidine tag were purchased from Qiagen (www.qiagen.com).

Coimmunoprecipitation experiments were performed on transfected COS-1 cells as described previously. ${ }^{11}$

The effect of tuberin expression on S6 phosphorylation was assayed in homozygote TSC2 knockout mouse embryo fibroblasts kindly provided by $\mathrm{H}$ Onda, Boston, USA. The cells were transfected with the wild type or G1556S TSC2 expression construct using lipofectAMINE PLUS reagent, according to the manufacturer's guidelines (Invitrogen, Carlsbad, CA, USA). Twenty four hours after transfection the medium was replaced with serum free medium and the cells incubated for an additional 24 hours before being processed for double label fluorescent microscopy, according to the recommended protocol (Cell Signaling Technology). Transfected cells expressing tuberin and showing inhibition of S6 phosphorylation were counted in three separate experiments, according to established methods. ${ }^{3}$ At least 50 cells were counted per experiment.

\section{RESULTS}

\section{Clinical findings}

A pedigree of the family is given in fig 1 , and the clinical symptoms of the mutation carriers are listed in table 1 . In the index patient (IV-3), fetal sonography revealed a cardiac "white spot" in the 22nd week of gestation, possibly due to the presence of a cardiac rhabdomyoma. Fourteen weeks later, three intracardial tumours of up to $5 \mathrm{~mm}$ in size were recorded. After birth these tumours were confirmed as $9 \times 6 \mathrm{~mm}$ and $8 \times 6 \mathrm{~mm}$ in size. Cerebral structures were normal and postnatal development was uncomplicated. No other cardiac abnormalities were reported. At the age of 2 months, clinical examination including Woods light revealed three typical hypomelanotic macules, sufficient for the diagnosis of TSC. Ophthalmological examination and CT were normal. The child is now 3 years of age and has attained normal developmental landmarks. Seizures have never been observed.

The sibling of the index patient (IV-2) was delivered by vacuum extraction after an uncomplicated pregnancy. At the age of 2 years he had one single febrile convulsion. He never received anticonvulsive medical treatment. Mental and speech development were slow. Clinical examination including Woods light at the age of 4 years revealed no cutaneous TSC symptoms. Cerebral MRT and CT as well as echocardiography were normal. At the age of 7 years an attention deficit hyperactivity disorder (ADHD) was diagnosed and treatment with Ritalin ${ }^{\circledR}$ (methylphenidat) was initiated. At that time he had remained free of seizures and displayed no cutaneous signs of TSC. This individual does not meet the diagnostic criteria for TSC.

Individual III-3, the father of IV-2 and IV-3, is 35 years of age and healthy. Clinical examination including Woods light revealed a single hypomelanotic macule of coin size on the left buttock. Renal ultrasound revealed bilateral local increases in echogeneity of the upper poles and a single echogenic structure of $1 \mathrm{~cm}$ in size, compatible with an angiomyolipoma. Liver sonography and cerebral CT were normal.

Individual III-2, the paternal aunt of the index patient, had been examined at the age of 20 years because of multiple depigmented lesions and suspected TSC. CT, eye fundus and nails were found to be normal. Examination at the age of 36 years revealed multiple characteristic hypomelanotic macules, but no complaints. She had a minor shagreen patch in the lumbar region of $2 \mathrm{~mm}$ in size, consistent with TSC.
Her son, individual IV-1, was admitted to hospital at the age of 11 years because of epileptic attacks which ceased after anticonvulsive treatment. EEG showed a focal activity of both temporoparietal regions and MRT revealed an increase in signal intensity in the meseal temporal lobes, which were interpreted as beginning hippocampal sclerosis, although inflammation or an astrocytoma could not be excluded at that time. A causal relationship of this finding to TSC is neither proven nor excluded. There were no cortical tubera and no calcified subependymal nodules. Sonography revealed a single kidney on the right. Eye fundi were normal. Dermatological examination at the age of 12 years revealed three hypomelanotic macules of up to $1.5 \mathrm{~cm} \times 2.5 \mathrm{~cm}$ in size.

Individual II-2, the grandfather of the index patient, was not available for examination. However, there were no reports of epileptic seizures or facial angiofibromas or any other TSC symptoms. Individual II-1 died at the age of 30 years from renal bleeding. The possibility that the bleeding was due to a renal angiomyolipoma was not excluded.

\section{Genetic analysis}

Complete sequencing of the coding regions of both TSC genes from DNA of peripheral leukocytes in the index patient (IV-3) identified a germline mutation $4684 \mathrm{G}>\mathrm{A}$ in the TSC2 gene (nucleotide numbering is as originally described ${ }^{15}$ and therefore does not include the 69 nucleotides of the alternatively spliced exon 31). No other sequence changes were identified at either the TSCl or TSC2 gene loci in the index patient.

Subsequent analysis of exon 36 of the TSC2 gene in the rest of the family identified the $4684 \mathrm{G}>\mathrm{A}$ substitution in individuals III-3, III-2, and IV-1. The TSC2 4684G $>$ A mutation was also suspected in individual II-2 who was not available for testing, and possibly individual II-1 who died from renal bleeding, consistent with the presence of a renal angiomyolipoma. The TSC2 4684G $>$ A substitution was excluded in individuals IV-2, III-4, and II-3.

To determine whether the TSC2 4684G $>$ A substitution represented a simple polymorphism, we screened exon 36 in an additional 50 control DNAs isolated from individuals without TSC. None of the individuals tested was positive for the $4684 \mathrm{G}>\mathrm{A}$ substitution. In addition, the $4684 \mathrm{G}>\mathrm{A}$ substitution was not detected in $>500$ TSC patients tested in Rotterdam, Erlangen, and Martinsried and, as far as we are aware, has not been described elsewhere in the literature.

To exclude the possibility of an undetected TSC 1 mutation in this family, linkage analysis was performed using four microsatellite markers, D9S2126, D9S1830, D9S1199, and D9S1198, flanking the TSCl gene. Individuals III-2, III-3, IV-1, and IV-3 did not share a common haplotype (data not shown). In contrast, linkage analysis using the markers KG8, 16AC2.5, and SM7, flanking the TSC2 gene, revealed a common haplotype in all mutation carriers, but not in the unaffected individual IV:2 (fig 1). For individual II:2, the same haplotype as in the mutation carriers could be reconstructed, supporting the hypothesis that the G1556S mutation was inherited from the grandfather.

\section{Functional analysis}

Coimmunoprecipitation and Western blot experiments were performed on COS-1 cells transfected with a TSC2 expression construct containing the $4684 \mathrm{G}>\mathrm{A}$ substitution. The cDNA used in these experiments lacks the 23 amino acids encoded by the alternatively spliced exon 31 , and this tuberin variant is therefore referred to as G1556S. The functional significance of the amino acids encoded by exon 31 appears minimal. ${ }^{16}$ Previous work had indicated that the majority of TSC2 mRNA transcripts do not contain the sequence corresponding to exon $31^{17}{ }^{18}$ and that the function of tuberin is not affected by the presence or absence of this sequence in the assays used 


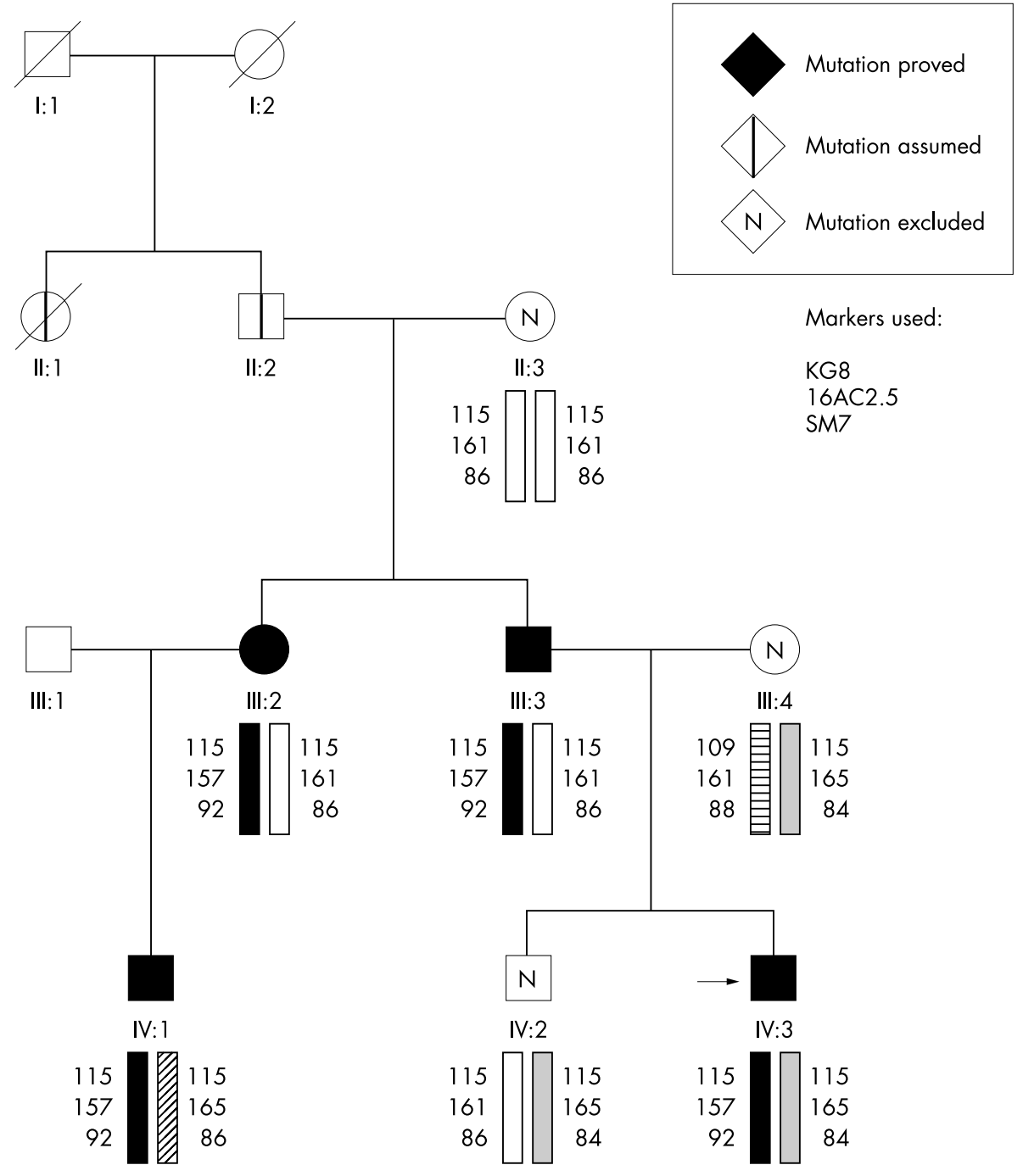

Figure 1 Segregation analysis of the G1556S mutation in the family confirming linkage to TSC2 by identical haplotypes in all mutation carriers.

here $^{12} ;$ ( $\mathrm{M}$ Nellist, unpublished data). The transfection experiments were repeated at least three times with the same results. Representative examples are shown in fig 2.

\section{Expression of the $\mathrm{G} 1556 \mathrm{~S}$ tuberin variant}

As shown in fig 2A, wild type tuberin migrates as a broad band on SDS-PAGE gels. This is due to the presence of

Table 1 Summary of clinical symptoms specific for TSC in the mutation carriers

\begin{tabular}{lllll}
\hline TSC trait & III-2 & III-3 & IV-1 & IV-3 \\
\hline Age at examination & 36 years & 35 years & 11 years & 2 months \\
Facial angiofibroma & - & - & - & - \\
Hypomelanotic macules* & + & $(+)$ & + & + \\
Shagreen patch & (+) & - & - & - \\
Ungual fibromas & - & - & - & - \\
Retinal hamartoma & - & - & - & n. e.f \\
Cerebral tubera & - & - & - & - \\
Subependymal nodules & - & - & - & n. e. \\
Epileptic seizures & - & - & + & - \\
Cardiac rhabdomyoma & n. e. & n. e & n. e. & + \\
Renal cysts or & - & + & - & n. e. \\
hamartomas & & & & \\
\hline
\end{tabular}

${ }^{*}(+),<3 ; \dagger(+)$, size $2 \mathrm{~mm}$; $\ddagger$ n.e., not examined; $\S$, one tumour of $1 \mathrm{~cm}$ size. differentially phosphorylated tuberin isoforms. ${ }^{19}$ The tuberin G1556S variant, however, migrated as a single high mobility band, consistent with a reduction in tuberin phosphorylation. Similar effects have been observed for other tuberin missense variants. ${ }^{11}$ Immunofluorescence microscopy indicated that there were no differences in the transfection efficiencies of the wild type compared with the G1556S tuberin constructs. Identical results were obtained using different preparations of both the wild type and G1556S variant expression constructs (data not shown).

As shown in fig 2B, coexpression of an active $\mathrm{PKB}$ isoform with the G1556S variant resulted in a shift to the low mobility, phosphorylated tuberin isoforms, similar to the wild type protein but distinct from another tuberin variant (R611Q) that is completely resistant to PKB dependent phosphorylation. Compared with either wild type tuberin or the R611Q variant, the changes in the mobility of the G1556S variant upon coexpression of PKB indicates that, although the G1556S variant is less susceptible to phosphorylation by $\mathrm{PKB}$ than wild type tuberin, it is not completely resistant to $\mathrm{PKB}$, in contrast to the R611Q variant.

Phosphorylation of wild type tuberin and the G1556S variant by PKB was confirmed using an antibody specific for the phosphorylated PKB target sequence. The phosphorylation resistant, high mobility R611Q tuberin variant was not detected with the PKB target sequence antibody. 
A Interaction with hamartin

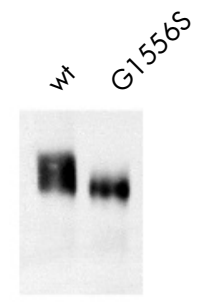

Lysate

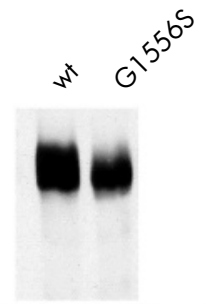

Tuberin IP

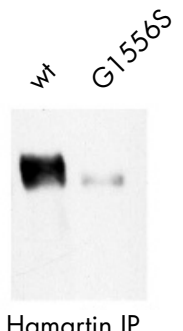

Hamartin IP
B PKB-dependent phosphorylation

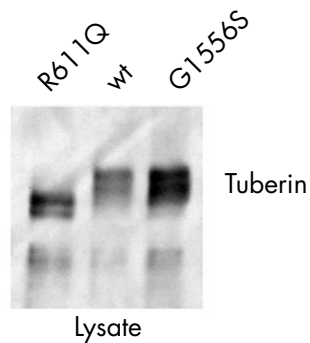

Lysate

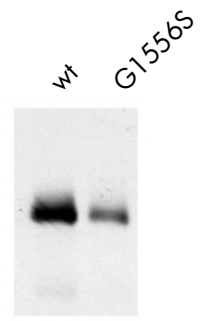

Lysate

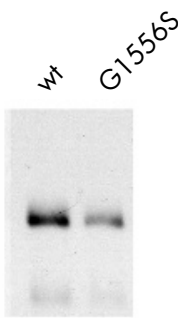

Tuberin IP

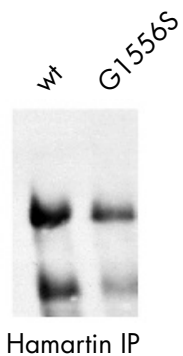

Hamartin IP

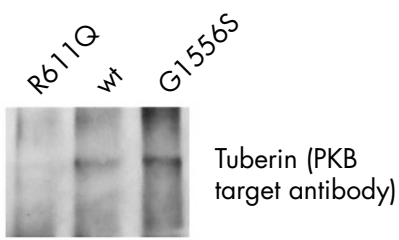

Tuberin IP

C Inhibition of S6 phosphorylation

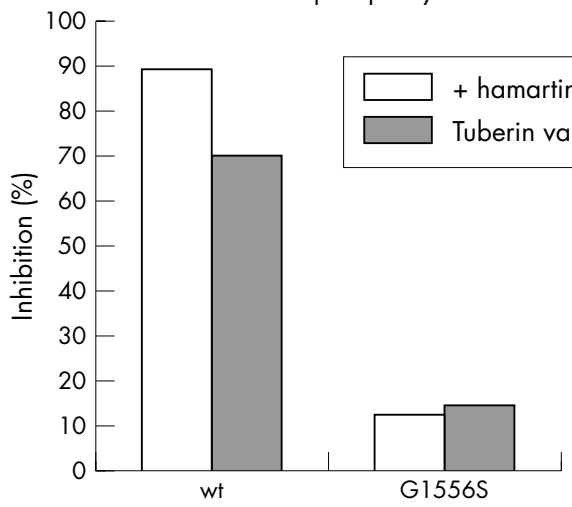

Figure 2 Functional comparison of wild type tuberin with the G1556S variant.(A) Interaction with hamartin. Cotransfected COS- 1 cells expressing hamartin and either wild type tuberin (wt) or the G1556S variant (G1556S) were lysed and the tuberin-hamartin complex was immunoprecipitated using antibodies specific for tuberin (Tuberin IP) and antibodies specific for hamartin (Hamartin IP). The amounts of (co)immunoprecipitated tuberin and hamartin were estimated by Western blotting. The examples shown are representative of at least three separate experiments. (B) PKB dependent phosphorylation. Cotransfected COS-1 cells expressing an activated isoform of PKB and either wild type tuberin (wt), the tuberin R611Q variant (R61 1Q), or the G1556S variant (G1556S) were lysed and analysed by Western blotting with an antibody specific for tuberin (lysate, above). Tuberin phosphorylated by PKB was detected by immunoprecipitation of tuberin, followed by incubation with the PKB target sequence antibody (Cell Signaling Technology) (Tuberin IP, below). The examples shown are representative of at least three separate experiments. (C) Inhibition of phosphorylation. TSC2 -/- MEFs were transfected with either the wild type TSC2 expression construct or the G1556S variant expression construct. After 24 hours the cells were serum starved for 24 hours prior to analysis by double label fluorescent microscopy. Transfected cells expressing wild type tuberin (or the G1556S variant) and either positive or negative for phosphorylated S6 were counted. The proportion of transfected cells without S6 phosphorylation is indicated.

\section{Interaction between hamartin and the tuberin G1556S variant}

Tuberin forms a complex with hamartin, the product of the TSCl gene, ${ }^{14}$ and the formation of the complex stabilises both proteins. ${ }^{20}$ To investigate whether the tuberin G1556S variant was able to interact with hamartin, coimmunoprecipitation experiments were performed. Hamartin and the tuberin G1556S variant could be coimmunoprecipitated. However, as shown in fig $2 \mathrm{~A}$, using antibodies specific for tuberin less hamartin was coimmunoprecipitated with the G1556S variant than with wild type tuberin and, using antibodies specific for hamartin, compared with wild type tuberin less of the G1556S variant was coimmunoprecipitated. Therefore, although complex formation was not abrogated completely, the G1556S substitution reduced the stability of the tuberinhamartin interaction.

Tuberin is necessary to maintain hamartin in a soluble form in the cytosol. ${ }^{20}$ The reduction in the amount of hamartin in the lysate of the cells expressing the G1556S tuberin variant is therefore consistent with the reduction in stability of the tuberin-hamartin complex caused by the G1556S substitution. 


\section{Inhibition of S6 phosphorylation by the tuberin G1556S variant}

The tuberin-hamartin complex inhibits the activation of S6 kinase, thereby preventing phosphorylation of the ribosomal protein S6, ${ }^{16}$ and tuberin missense mutations have been shown to prevent this inhibition. ${ }^{3}$ The effect of the tuberin G1556S variant on S6 phosphorylation was investigated in mouse embryo fibroblasts (MEFs) lacking endogenous tuberin using an established assay. ${ }^{3}$ As shown in fig $2 \mathrm{C}$, no S6 phosphorylation was visible in $70 \%$ of the cells expressing wild type tuberin. Co-expression of hamartin resulted in inhibition of S6 phosphorylation in almost $90 \%$ of the transfected cells. In contrast, the inhibition of S6 phosphorylation by the G1556S variant, either expressed alone or co-expressed with hamartin, was reduced approximately fourfold. Less than $20 \%$ of the cells expressing the G1556S variant showed inhibition of S6 phosphophorylation.

\section{DISCUSSION}

We report on the identification of a novel TSC2 missense mutation, 4684G $>$ A (G1556S), in a patient with the clinical diagnosis of TSC due to prenatal cardiac rhabdomyomas and the development of hypomelanotic macules in the first months of life. Subsequent analysis of the parents of the index patient and further family members to distinguish between a disease causing mutation and a non-pathogenic polymorphism identified three additional individuals with the $4684 \mathrm{G}>$ A substitution. Although only the index patient fulfilled the established criteria for the diagnosis of TSC, ${ }^{6}$ the other family members with the $4684 \mathrm{G}>\mathrm{A}$ substitution all displayed minor signs of TSC, preferentially hypomelanotic macules (table 1). None of them had cortical tubera or calcifications and only one (IV-1) suffered from epileptic seizures suspected to result from a hippocampus sclerosis. These findings are so far consistent with the hypothesis that the $4684 \mathrm{G}>\mathrm{A}$ substitution is indeed a pathogenic mutation. No other changes were identified in either the TSC2 gene or the TSCl gene of the index patient. Analysis of microsatellite markers across both loci excluded linkage to TSCl, but confirmed identical haplotypes for TSC2 in all mutation carriers. The possibility of a second undetected mutation on the same allele as the $4684 \mathrm{G}>\mathrm{A}$ substitution, although not excluded, seems unlikely.

The $4684 \mathrm{G}>\mathrm{A}$ mutation leads to a change of glycine, a non-polar residue which is completely conserved between human, mouse, rat, Fugu and Drosophila (fig 3) for serine, a polar amino acid in the C-terminal domain of tuberin.

Several regions with possible functional importance have been reported for tuberin: the hamartin binding region at the $\mathrm{N}$-terminus, ${ }^{14}{ }^{21}$ the putative raplGAP homology region with GAP activity for $\operatorname{Rapl}^{22}$ and Rab5, ${ }^{23}$ two transcriptional activating domains, ${ }^{24}$ as well as binding regions for Rabaptin5, ${ }^{23}$ steroid hormone receptors, ${ }^{25}$ and Calmodulin. ${ }^{26}$ The latter are all located in the C-terminal domain which has been shown to be important for growth suppression. ${ }^{27}$

Only a few tuberin missense changes have been tested for their ability to prevent formation of the tuberin-hamartin complex $^{1011}$ or inhibit S6K activation. ${ }^{3512}$ Amino acid

$\begin{array}{ll}\text { Human } & \text { ILSNEHGSYRYTEE LTGLGRLIELKDCQPD } \\ \text { Mouse } & \text { ILSNEHGSYRYTEF LTGLGRLIELKDCQPD } \\ \text { Rat } & \text { ILSNEHGSYRYTEE LTGLGRLIELKDCQPD } \\ \text { Fugu } & \text { ILSNEYGSNRYAAF LTGLGKLIHLKDCDPD } \\ \text { Drosophila } & \text { ILRNSHGSARYVEE LRNIGTLVSLKEAEQN }\end{array}$

Figure 3 Evolutionary conservation of TSC2 exon 36. Identical amino acids are shaded in grey. The G1556 residue affected by the mutation is additionally highlighted in bold. changes in different regions of tuberin, outside the predicted phoshorylation sites and putative hamartin binding domain, have been shown to regulate tuberin phosphorylation and the tuberin-hamartin interaction, possibly through conformational changes. ${ }^{11}{ }^{19}$ Some tuberin missense mutations that do not prevent tuberin-hamartin binding are still unable to inhibit S6K phosphorylation. ${ }^{10}{ }^{12}$

To determine whether the $4684 \mathrm{G}>\mathrm{A}$ substitution is a pathogenic mutation, the effects of the replacement of the glycine residue with serine on tuberin function were investigated. Compared with wild type tuberin, the G1556S tuberin variant was hypophosphorylated, reduced in its ability to form a stable complex with hamartin, and compromised in its ability to inhibit S6 phosphorylation. Therefore, in all the assays of tuberin function the G1556S variant was impaired, consistent with the TSC2 4684G>A substitution being the pathogenic mutation in this family.

Despite the clear functional deficiencies of the tuberin G1556S variant, the phenotype in this family was mild. One possible explanation for this is that the G1556S variant retains some activity. Some other mutations, such as the tuberin R611Q substitution, for example, are not phosphorylated and are unable to form a complex with hamartin. ${ }^{11}$ It will be interesting to compare the phenotypes of TSC patients with TSC2 missense mutations that have different effects on tuberin phosphorylation, complex formation, and the inhibition of S6K.

In summary, during routine mutation screening in TSC patients we identified a new sequence variation $4684 \mathrm{G}>\mathrm{A}$ in the TSC2 gene with unknown pathological significance. Analysis of the family of the index patient identified three additional individuals with the same sequence change who all exhibited minor clinical signs of TSC. Alone, none of these additional individuals fulfilled the diagnostic criteria for TSC, and none of them would have been diagnosed with TSC if they were not related to the index patient. However, functional analysis of the tuberin G1556S variant showed that the mutant protein was hypophosphorylated with respect to the wild type protein, that the stability of the interaction between hamartin and the variant tuberin was reduced relative to the wild type tuberin-hamartin complex, and that the substitution prevented tuberin dependent inhibition of S6 phosphorylation.

These findings confirm the pathogenicity of TSC2 $4684 \mathrm{G}>\mathrm{A}$ and are consistent with the mild clinical phenotype in the mutation carriers in this family. The approach described here will help to define which domains and amino acid residues are critical for tuberin function and provide insight into the possibility that additional phenotypegenotype correlations exist in TSC. Moreover, the family presented here highlights the necessity for careful and complete clinical evaluation of both apparently affected and unaffected members in families with TSC.

\section{ACKNOWLEDGEMENTS}

The authors would like to thank the family members for their readiness to participate in this study. Dr H Onda is thanked for kindly providing the TSC2 -/- mouse embryo fibroblasts and Dr AMW van den Ouweland is thanked for critical reading of the manuscript. Financial support for M Goedbloed and M Nellist was provided by Noortman B.V., the Michelle Foundation, and the Nederlandse Organisatie voor Wetenschappelijk Onderzoek.

\section{Authors' affiliations}

K Mayer, Laboratory for Medical Genetics, Lochhamer Str. 29, 82152 Martinsried, Germany

M Goedbloed, K van Zijl, M Nellist, Department of Clinical Genetics, Erasmus MC, Dr. Molewaterplein 50, 3015 GE Rotterdam, The Netherlands 
H-D Rott, Institute of Human Genetics, University Erlangen-Nuremberg, Schwabachanlage 10, 91054 Erlangen, Germany

Conflicts of interest: none declared

Correspondence to: Dr K Mayer, Laboratory for Medical Genetics, Lochhamer Str. 29, 82152 Martinsried, Germany;

mayer@medizinische-genetik.de

Revised version received 14 August 2003

Accepted for publication 1 September 2003

\section{REFERENCES}

1 Marygold SJ, Leevers SJ. Growth Signaling: TSC Takes Its Place. Curr Biol 2002;12(22):R785-7

2 McManus EJ, Alessi DR. TSC1-TSC2: a complex tale of PKB-mediated S6K regulation. Nat Cell Biol 2002;4(9):E214-16.

3 Jaeschke A, Hartkamp J, Saitoh M, Roworth W, Nobukuni T, Hodges A, Sampson J, Thomas G, Lamb R. Tuberous sclerosis complex tumor suppressormediated S6 kinase inhibition by phosphatidylinositide-3-OH kinase is mTOR independent. J Cell Biol 2002;159(2):217-24.

4 Gao X, Pan D. TSC1 and TSC2 tumor suppressors antagonize insulin signaling in cell growth. Genes Dev 2001;15(11):1383-92.

5 Tee AR, Fingar DC, Manning BD, Kwiatkowski DJ, Cantley LC, Blenis J. Tuberous sclerosis complex-1 and -2 gene products function together to inhibit mammalian target of rapamycin (mTOR)-mediated downstream signaling. Proc Natl Acad Sci U S A 2002;99(21):13571-6.

6 Roach ES, DiMario FJ, Kandt RS, Northrup H. Tuberous Sclerosis Consensus Conference: recommendations for diagnostic evaluation. National Tuberous Sclerosis Association. J Child Neurol 1999;14(6):401-7.

7 Dabora SL, Jozwiak S, Franz DN, Roberts PS, Nieto A, Chung J, Choy YS, Reeve MP, Thiele E, Egelhoff JC, Kasprzyk-Obara J, Domanska-Pakiela D, Kwiatkowski DJ. Mutational analysis in a cohort of 224 tuberous sclerosis patients indicates increased severity of TSC2, compared with TSC1, disease in multiple organs. Am J Hum Genet 2001;68(1):64-80.

8 Jones AC, Daniells CE, Snell RG, Tachataki M, Idziaszczyk SA, Krawczak M, Sampson JR, Cheadle JP. Molecular genetic and phenotypic analysis reveals differences between TSC1 and TSC2 associated familial and sporadic tuberous sclerosis. Hum Mol Genet 1997:6(12):2155-61.

9 Langkau N, Martin N, Brandt R, Zugge K, Quast S, Wiegele G, Jauch A, Rehm M, Kuhl A, Mack-Vetter M, Zimmerhackl LB, Janssen B. TSC1 and TSC2 mutations in tuberous sclerosis, the associated phenotypes and a model to explain observed TSC1/TSC2 frequency ratios. Eur J Pediatr 2002;161(7):393-402.

10 Hodges AK, Li S, Maynard J, Parry L, Braverman R, Cheadle JP, DeClue JE, Sampson JR. Pathological mutations in TSC1 and TSC2 disrupt the interaction between hamartin and tuberin. Hum Mol Genet 2001;10(25):2899-905.

11 Nellist M, Verhaaf B, Goedbloed MA, Reuser AJ, van den Ouweland AM, Halley DJ. TSC2 missense mutations inhibit tuberin phosphorylation and prevent formation of the tuberin-hamartin complex. Hum Mol Genet 2001;10(25):2889-98

12 Inoki K, Li Y, Zhu T, Wu J, Guan KL. TSC2 is phosphorylated and inhibited by Akt and suppresses mTOR signalling. Nat Cell Biol 2002;4(9):648-57.

13 Maheshwar MM, Cheadle JP, Jones AC, Myring J, Fryer AE, Harris PC Sampson JR. The GAP-related domain of tuberin, the product of the TSC2 gene, is a target for missense mutations in tuberous sclerosis. Hum Mol Genet 1997;6(11):1991-6.

14 van Slegtenhorst $M$, Nellist $M$, Nagelkerken B, Cheadle J, Snell R, Van Den OA, Reuser A, Sampson J, Halley D, van der SP. Interaction between hamartin and tuberin, the TSC1 and TSC2 gene products. Hum Mol Genet 1998;7(6): 1053-7.

15 Identification and characterization of the tuberous sclerosis gene on chromosome 16. The European Chromosome 16 Tuberous Sclerosis Consortium. Cell 1993;75(7):1305-15.

16 Kwiatkowski DJ. Tuberous Sclerosis: from Tubers to mTOR. Ann Hum Genet 2003;67:87-96

17 Xiao GH, Jin F, Yeung RS. Identification of tuberous sclerosis 2 messenger RNA splice variants that are conserved and differentially expressed in rat and human tissues. Cell Growth Differ 1995;6(9): 1185-91.

18 Xu L, Sterner C, Maheshwar MM, Wilson PJ, Nellist M, Short PM, Haines JL, Sampson JR, Ramesh V. Alternative splicing of the tuberous sclerosis 2 (TSC2) gene in human and mouse tissues. Genomics 1995;27(3):475-80.

19 Aicher LD, Campbell JS, Yeung RS. Tuberin phosphorylation regulates its interaction with hamartin. Two proteins involved in tuberous sclerosis. J Biol Chem $2001 ; 276(24): 21017-21$.

20 Nellist $M$, van Slegtenhorst MA, Goedbloed $M$, van den Ouweland AM, Halley DJ, van der SP. Characterization of the cytosolic tuberin-hamartin complex. Tuberin is a cytosolic chaperone for hamartin. J Biol Chem $1999 ; 274(50): 35647-52$

21 Plank TL, Yeung RS, Henske EP. Hamartin, the product of the tuberous sclerosis 1 (TSC1) gene, interacts with tuberin and appears to be localized to cytoplasmic vesicles. Cancer Res 1998;58(21):4766-70.

22 Wienecke R, Konig A, DeClue JE. Identification of tuberin, the tuberous sclerosis-2 product. Tuberin possesses specific Rap IGAP activity. J Biol Chem 1995;270(27): 16409-14.

23 Xiao GH, Shoarinejad F, Jin F, Golemis EA, Yeung RS. The tuberous sclerosis 2 gene product, tuberin, functions as a Rab5 GTPase activating protein (GAP) in modulating endocytosis. J Biol Chem 1997;272(10):6097-100.

24 Tsuchiya H, Orimoto K, Kobayashi K, Hino O. Presence of potent transcriptional activation domains in the predisposing tuberous sclerosis (Tsc2) gene product of the Eker rat model. Cancer Res 1996;56(3):429-33.

25 Henry KW, Yuan X, Koszewski NJ, Onda H, Kwiatkowski DJ, Noonan DJ. Tuberous sclerosis gene 2 product modulates transcription mediated by steroid hormone receptor family members. J Biol Chem 1998;273(32):20535-9.

26 Noonan DJ, Lou D, Griffith N, Vanaman TC. A calmodulin binding site in the tuberous sclerosis 2 gene product is essential for regulation of transcription events and is altered by mutations linked to tuberous sclerosis and lymphangioleiomyomatosis. Arch Biochem Biophys 2002;398(1):132-40.

27 Momose S, Kobayashi T, Mitani H, Hirabayashi M, Ito K, Ueda M, Nabeshima $Y$, Hino $O$. Identification of the coding sequences responsible for $\mathrm{Tsc} 2$-mediated tumor suppression using a transgenic rat system. Hum Mol Genet 2002; 11(24):2997-3006 